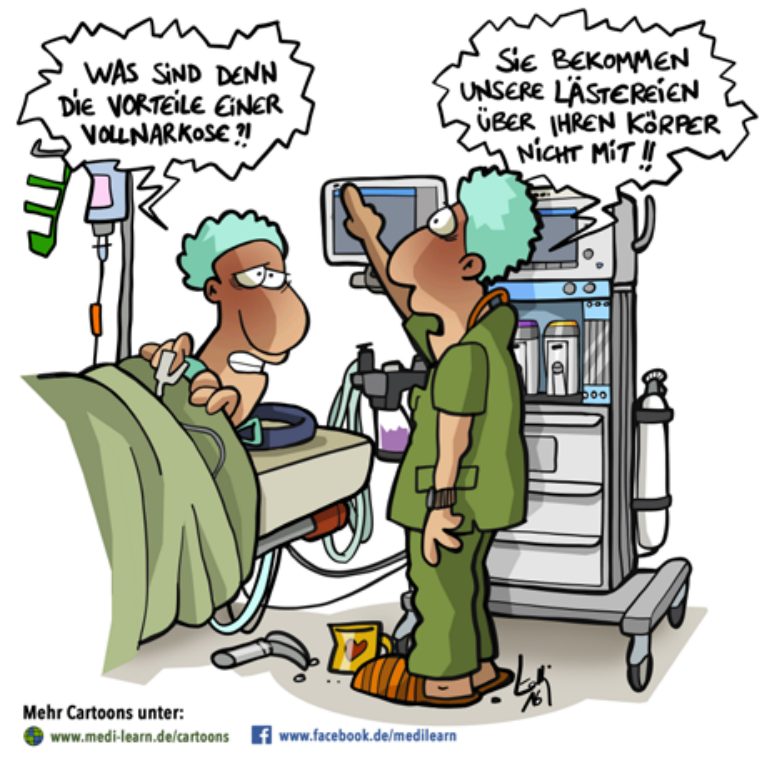

„Mehr Praxisbezug im Studium und eine Stärkung der Allgemeinmedizin sind gerade mit Blick auf die gute Versorgung im ländlichen Raum von großer Bedeutung."

Bundesgesundheitsminister Hermann Gröhe zum „Masterplan Medizinstudium 2020"

\title{
Angebohrt
}

\section{Füllung aus der Steinzeit}

Dass der Steinzeitmensch Ötzi unter Karies und Parodontitis gelitten haben muss, ist hinlänglich bekannt. Wir fragen uns: War er vielleicht auf dem Weg zu seinem Zahnarzt nach Italien, als er vor etwa 5250 Jahren sein Leben in den Südtiroler Alpen ließ? Archäologen von der University of Bologna haben jedenfalls 13.000 Jahre alte Schneidezähne gefunden, die bereits zahnärztliche Behandlungen aufwiesen - Bohren und auch Füllen war also schon in der Steinzeit möglich.

Mithilfe kleiner Steinutensilien meißelten unsere geschickten Urahnen an den Zähnen ihrer Zeitgenossen. Da wurde mit dem Steinzeitbohrer ein Loch ausgebohrt und der freiliegende Wurzelkanal mit einer Mischung aus dem teerartigen Bitumen, Pflanzenfasern und Haaren zur Desinfektion und Schmerzstillung abgedichtet. Wir vermuten: Der Begriff „Haare auf den Zähnen haben“ stammt dann wohl aus dieser Zeit.

\section{Vitamin aus der Zahnpasta}

Etwa 900.000 Menschen leben in Deutschland vegan. Nun soll vegane Ernährung ja durchaus ökologisch sinnvoll sein, allerdings steigt bei veganer und übrigens auch bei vegetarischer Lebensweise das Risiko, zu wenig Vitamin B12 aufzunehmen, da dieses fast ausschließlich in tierischer Nahrung zu finden ist.

Solch ein Vitamin-B12-Mangel bringt Symptome wie Müdigkeit, Konzentrationsstörungen oder Kopfschmerzen mit sich und kann bis zu Lähmungserscheinungen führen. Doch Rettung naht - und es muss ja nicht immer eine Pille oder gleich eine (Vitamin-)Spritze sein. Nein, Rettung kommt aus der Zahnmedizin, ausgerechnet aus einer Zahnpasta, die mit Vitamin B12 angereichert wurde. Laut des „American Journal of Clinical Nutrition” erhielt eine Gruppe das mit Vitamin B12 angereicherte Zahngel, die andere Gruppe ein Präparat ohne den Zusatz. Nach zwölf Wochen hatte die Verwendung des Gels den Vitamin-B12-Status der Veganer tatsächlich verbessert. Wer nun glaubt, die Veganer hätten vor lauter Hunger das Gel gegessen: Das Vitamin wurde diskret über die Mundschleimhaut aufgenommen. Gut zu wissen: Eine Überdosierung von Vitamin B12 ist kaum möglich. 